\title{
Las Compuestas endémicas de Oaxaca, México: DIVERSIDAD Y DISTRIBUCIÓN
}

\author{
Mario ERnesto SuÁrez-Mota ${ }^{1}$ y José Luis Villaseñor ${ }^{2,3}$ \\ 'Facultad de Estudios Superiores Iztacala, Universidad Nacional Autónoma de México \\ ${ }^{2}$ Instituto de Biología, Universidad Nacional Autónoma de México \\ ${ }^{3}$ Autor para correspondencia: vrios@ibiologia.unam.mx
}

\begin{abstract}
Resumen: Se reconocen 249 especies de la familia Asteraceae (Compositae) como endémicas o casi endémicas de Oaxaca, al distribuirse marginalmente en alguno de los estados con los que Oaxaca hace frontera. Oaxaca comparte el mayor número de especies endémicas con Puebla (53.4\%), mientras que con Veracruz comparte el menor número (2.8\%). Se analiza la distribución de 209 especies que contaron con registros georreferenciados, en una retícula de 162 cuadros de 15 minutos de latitud y longitud en que fue dividido el estado; los resultados indican que 103 de ellos (63.4\%) registran al menos una especie endémica. Al evaluar la distribución del endemismo en una categorización de cinco tipos principales de vegetación (biomas), se observa que los bosques templados registran la mayor riqueza de especies (164) y los bosques tropicales húmedos la menor riqueza (15 especies). Se discuten las principales áreas de concentración de especies endémicas en el estado y su relación con los biomas donde estas especies se encuentran.
\end{abstract}

Palabras clave: Asteraceae, biomas, endemismo, México, Oaxaca

\begin{abstract}
A figure of 249 species of the family Asteraceae (Compositae) endemic or nearly endemic to the state of Oaxaca are identified, several of them marginally distributed at the neighbor states bordering Oaxaca. The state shares the larger number of endemic species with Puebla (53.4\%), while the lower number is shared with the state of Veracruz (2.8\%). This paper analyses the geographic distribution of 209 species of endemic Asteraceae with specimens that were able to be georreferenced, using a grid square network with each cell 15 minutes of latitude and longitude ( $\mathrm{N}=162$ grid squares). Results indicate that 103 grid squares (63.4\%) record at least one endemic species. In addition, by using a classification of the state in five main vegetation types (biomes), it is noted that temperate forests include the largest number of endemic species (164) and the tropical humid forests the lower number (15). The main areas in Oaxaca concentrating endemic species are discussed, searching for any relationship with the biomes such cells include.
\end{abstract}

Key words: Asteraceae, biomes, endemism, Mexico, Oaxaca

a biodiversidad no se distribuye de manera homogénea a Llo largo del planeta. Existen países o regiones que concentran importantes cifras de especies, actualmente identificados como megadiversos (Mittermeier, 1988; Akeroyd y Synge, 1992). Estas zonas de megadiversidad han sido propuestas como de interés especial para su protección por la alta riqueza de especies que albergan. Sin embargo, existe dentro del concepto de biodiversidad un componente más particular y que ha destacado también tanto por su relevancia biogeográfica como por su interés en la biología de la conservación: el endemismo.

Un taxón endémico es aquel que restringe su distribución a una región específica, ya sea natural (por ejemplo una provincia biogeográfica) o política (un país, un estado, etc.). El endemismo de una región representa la fracción exclusiva de su riqueza biológica; Pressey et al. (1993) destacaron esta exclusividad al introducir en la biología de la conservación el concepto de irremplazabilidad. Una región es irremplazable si contiene taxones (especies o cualquier otra jerarquía taxonómica) exclusivos (endémicos), no encontrados en ninguna otra región. En la actualidad, cualquier estrategia encaminada a la conservación de la diversidad biológica necesita considerar enfáticamente a los sitios con endemismos, pues su pérdida ocasionará también la extinción de dichos endemismos.

Las áreas de concentración de endemismos, además de importantes para la biología de la conservación (Goombridge, 1992; Davis et al., 1997; Reid, 1998; Myers et al., 2000; 
Krupnick y Kress 2003; Kier et al., 2005; Villaseñor et al., 2006), son relevantes en los estudios biogeográficos. Henderson (1991) las considera fundamentales en los estudios de biogeografía histórica; de hecho, la determinación de áreas de endemismo es para algunos autores uno de los objetivos más importantes de la biogeografía (Cracraft, 1982; Rosen, 1988; Morrone, 1994), por ser estas áreas probables sitios de origen y diversificación.

México es considerado entre los primeros cinco países megadiversos por su riqueza florística (Mittermeier, 1988; Mittermeier y Goettsch, 1992; Villaseñor, 2003); está igualmente ubicado entre los países con mayor nivel de endemicidad en su flora (Villaseñor, 2003). La flora endémica de México ha recibido atención, tanto a nivel nacional como regional; por ejemplo, Rzedowski (1991a) discute estos niveles en la flora de México, estimando en 52\% el porcentaje de especies endémicas del territorio nacional, cifra no muy distinta a 56.6\% propuesta por Villaseñor (2003). A nivel regional se discute en la Mixteca Oaxaqueña-Poblana (García-Mendoza et al., 1994), en la Sierra de Manantlán en los estados de Colima y Jalisco (Hernández, 1995), en la península de Baja California (Villaseñor y Elias, 1995), en la Faja Volcánica Transmexicana (Delgadillo et al., 2003; Alcántara y Paniagua, 2007; Villaseñor y Ortiz, 2007) y en la provincia florística del Valle de Tehuacán-Cuicatlán (Méndez-Larios et al., 2004). A nivel estatal o municipal se encuentran también algunos estudios sobre endemicidad, como los realizados en el municipio de Culiacán, Sinaloa (Vega et al., 2000) o en el estado de Veracruz (Castillo et al., 2005).

La familia Asteraceae (o Compositae) ocupa un lugar predominante en la flora de México (Villaseñor, 2003). Sus poco más de 3,000 especies la ubican como la familia más diversa, representando alrededor de $13.5 \%$ de la riqueza florística total del país. Esta familia a su vez registra poco más de la mitad de sus especies como endémicas de México (Turner y Nesom, 1993; Villaseñor, 1993, 2003) y constituye un referente obligado al hablar de la riqueza florística de México, tanto a nivel nacional como regional o estatal. Por ejemplo, la familia ha sido utilizada como taxón representativo para estimar patrones de riqueza de la flora nacional (Rzedowski, 1991b; Villaseñor et al., 2007). La discusión entonces sobre los patrones de riqueza y endemicidad de esta familia puede ayudar a entender los patrones de la riqueza florística en su conjunto, al utilizarla como substituto de la diversidad florística. Por otra parte, algunos patrones de endemicidad en la familia han sido identificados, por ejemplo en la Faja Volcánica Transmexicana (Villaseñor y Ortiz, 2007), en la Sierra Madre Oriental (González-Zamora et al., 2007) o en el estado de Oaxaca (Villaseñor et al., 2004).

Oaxaca es el estado mexicano con la mayor riqueza de Compuestas (Asteraceae); su número asciende a 858 especies, de las cuales $502(58.5 \%)$ son endémicas de México y $133(15.5 \%)$ son endémicas del estado (Villaseñor et al.,
2004). Por otra parte, el estado comparte 116 especies endémicas de México con sus estados vecinos (Chiapas, Guerrero, Puebla y Veracruz) lo que eleva la cifra de endémicas regionales a 249 . La alta proporción de especies endémicas conocidas en esta región del país, constituyen un conjunto importante de taxones para definir áreas de concentración de endemismos en el estado; por tal razón, los objetivos de este trabajo son determinar las áreas de concentración de especies endémicas o casi endémicas de Compuestas en el estado de Oaxaca y evaluar su distribución en los principales tipos de vegetación. El estudio es motivado por la urgente necesidad de progresar en la acumulación del conocimiento para una mejor detección de las áreas prioritarias para la conservación de la biodiversidad en Oaxaca.

\section{Materiales y método}

A partir de una lista de 502 especies de Compuestas endémicas de México y presentes en el estado de Oaxaca, se seleccionaron 249 cuya distribución geográfica conocida se restringe a Oaxaca y estados vecinos (Chiapas, Guerrero, Puebla y Veracruz). Para cada una de estas especies se revisaron críticamente los ejemplares de herbario depositados en diversos herbarios, tanto nacionales como del extranjero (ver agradecimientos) para validar su correcta identificación; la información acerca de los sitios de recolecta fueron traducidos a coordenadas geográficas y utilizando un sistema de información geográfica (SIG, Arc View 3.2) se elaboraron mapas de distribución para evaluar la distribución geográfica de las especies (Figura 1).

La Unión Mundial para la Naturaleza (UICN) considera que una buena estrategia de análisis de los patrones de distribución de las especies es mediante el uso de cuadros o celdas en que se divide a la región de estudio (UICN, 2001), especialmente cuando se pretende calcular las áreas donde

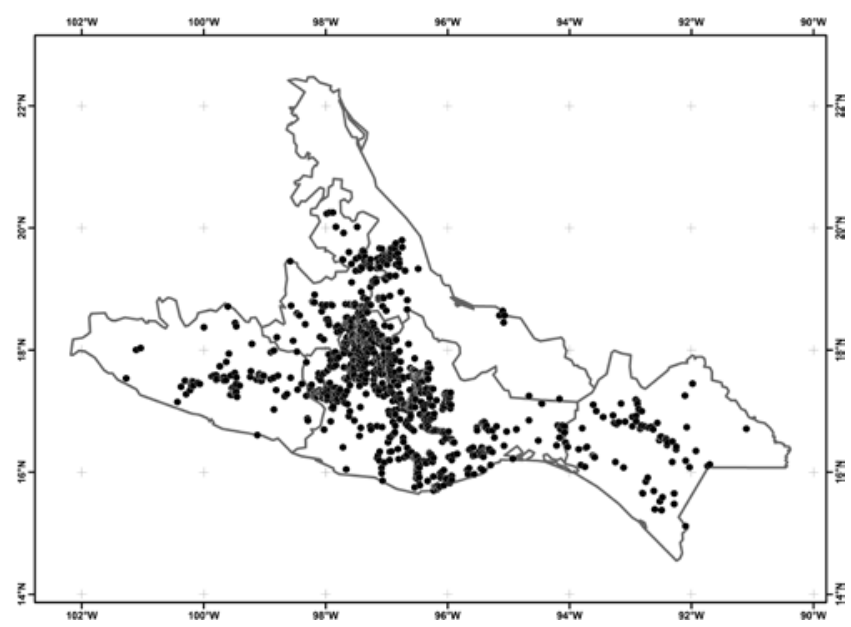

Figura 1. Localidades de recolecta de las Compuestas endémicas de México presentes en Oaxaca y estados vecinos. 


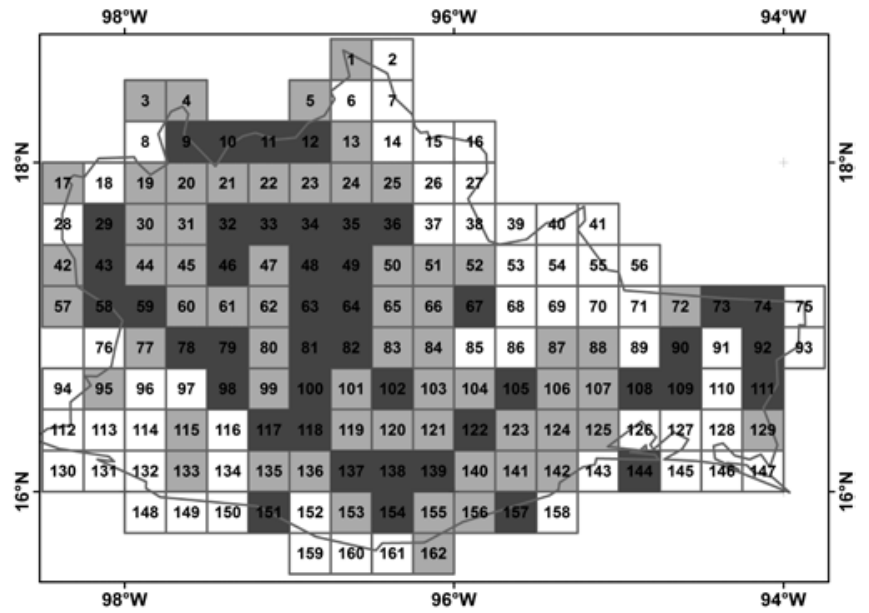

Figura 2. División del estado de Oaxaca en cuadros de 15 minutos de latitud y longitud. El número asignado a cada cuadro corresponde con su referencia a lo largo del texto y en el Apéndice 1. Los cuadros sombreados indican la presencia de especies de Compuestas endémicas del estado y endémicas compartidas con estados vecinos; el tono más obscuro señala las celdas con especies restringidas (conocidas solamente de una celda).

se encuentran ("extensión de la presencia" o "area of occupancy"). El tamaño de la cuadrícula fue determinada también siguiendo las recomendaciones de la UICN: para una especie en particular bajo análisis, el tamaño del cuadro o celda debe ser $10 \%$ de la distancia total de los puntos extremos conocidos para esta especie. En nuestro análisis, se calculó la distancia de los sitios extremos conocidos para cada especie $(\mathrm{N}=209$, pues para 40 especies no se contó con datos de localidad que pudieran ser georreferenciados); tomando el $10 \%$ de la distancia extrema de cada especie y promediando todos estos valores se obtuvo un valor que fue utilizado para determinar el tamaño de celda. Por tal razón, el estado de Oaxaca fue dividido en celdas de 15 minutos de latitud y longitud, pues la distancia promedio de todas las especies analizadas fue de $276.7 \mathrm{~km}$ y el $10 \%$ de dicho valor más o menos corresponde con una celda de 15 minutos de latitud y longitud (aproximadamente $27 \mathrm{~km}$ por lado). De esta manera, el estado de Oaxaca fue dividido en 162 cuadros (Figura 2). Una vez definido el tamaño de la retícula, una lista de las especies para cada cuadro se obtuvo intersectando en el SIG los mapas de distribución de las especies con el mapa de Oaxaca dividido en celdas.

La porción de Oaxaca del mapa de vegetación potencial de Rzedowski (1990) fue agrupada en cinco categorías (biomas): 1) bosque estacionalmente seco (BES, que incluye a los bosques espinosos y a los bosques tropicales caducifolios) 2) bosque húmedo (mesófilo) de montaña (BHM), 3) bosque templado (BTEM, que incluye a los bosques de coníferas y de encinos principalmente), 4) bosque tropical húmedo (BTHU, que incluye a los bosques tropicales peren- nifolios, subperennifolios y subcaducifolios) y 5) matorral xerófilo (MXE). Una vez hecha esta reclasificación, los sitios de recolecta (coordenadas geográficas) fueron ubicados en el mapa para definir su tipo de vegetación de acuerdo con el mapa reclasificado.

\section{Resultados}

En el estado de Oaxaca, 502 especies de Asteraceae, de un total de 858, son endémicas de México (Villaseñor et al., 2004). Por otra parte, 133 de las 502 especies restringen su distribución al territorio estatal (endémicas estrictas) y otras 116 solamente se conocen de Oaxaca y estados vecinos (Chiapas, Guerrero, Puebla y Veracruz). Entre las especies compartidas, 42 lo son con el estado de Puebla, 23 con Guerrero, 16 con Chiapas y siete con Veracruz. Solamente 28 especies se encuentran en Oaxaca y dos o más estados vecinos. El Cuadro 1 resume la distribución regional del endemismo presente en Oaxaca.

La figura 1 muestra la distribución de 209 de las 249 especies endémicas regionales que contaron con localidades georreferenciadas, 87 de ellas endémicas estrictas de Oaxaca. En total el mapa representa la ubicación de 2,104 localidades únicas (registros), que reportan a su vez una o más especies. La distribución de registros por especie no fue uniforme, pues 59 especies se ubicaron en el estado por un solo registro, mientras que solamente 20 de ellas tuvieron 25 o más registros.

Las 209 especies evaluadas geográficamente se incluyen en 80 géneros (Apéndice 1), siendo los géneros Ageratina y Verbesina los que registraron la mayor cantidad de especies endémicas (19 y 15 respectivamente). Otros géneros con una importante proporción de especies endémicas son Stevia (12), Acourtia (10), Roldana (9), Psacalium (8), Archibaccharis (6) o Viguiera (6). Por otra parte, 39 géne-

Cuadro 1. Distribución de las Compuestas endémicas de México presentes en Oaxaca y estados vecinos ( $N=209)$.

\begin{tabular}{lc}
\hline Distribución & Especies $(\%)$ \\
\hline Exclusivas de Oaxaca & $133(53.4)$ \\
Oaxaca-Puebla & $42(16.9)$ \\
Oaxaca-Guerrero & $23(9.2)$ \\
Oaxaca-Chiapas & $16(6.4)$ \\
Oaxaca-Veracruz & $7(2.8)$ \\
Oaxaca-Puebla-Veracruz & $9(3.6)$ \\
Oaxaca-Guerrero-Puebla & $7(2.8)$ \\
Oaxaca-Chiapas-Veracruz & $5(2.0)$ \\
Oaxaca-Chiapas-Guerrero & $4(1.6)$ \\
Oaxaca-Chiapas-Puebla & $1(0.4)$ \\
Oaxaca-Chiapas-Guerrero-Puebla & $1(0.4)$ \\
Oaxaca-Chiapas-Puebla-Veracruz & $1(0.4)$ \\
\hline
\end{tabular}


Cuadro 2. Número de especies endémicas de Oaxaca y estados vecinos en los cuadros o celdas en que fue dividido el estado. Solamente se indican las celdas que registraron especies endémicas. Entre paréntesis se indica el número de especies solamente conocidas (restringidas) a la celda.

\begin{tabular}{|c|c|c|c|c|c|}
\hline $\begin{array}{l}\text { Cuadro } \\
\text { o celda }\end{array}$ & Especies & $\begin{array}{l}\text { Cuadro } \\
\text { o celda }\end{array}$ & Especies & $\begin{array}{l}\text { Cuadro } \\
\text { o celda }\end{array}$ & Especies \\
\hline 1 & 2 & 42 & 2 & 77 & 2 \\
\hline 3 & 3 & 43 & $21(7)$ & 78 & $3(2)$ \\
\hline 4 & 11 & 44 & 17 & 79 & $1(1)$ \\
\hline 5 & 1 & 45 & 4 & 80 & 1 \\
\hline 9 & $33(2)$ & 46 & $17(3)$ & 81 & $22(3)$ \\
\hline 10 & $35(3)$ & 47 & 13 & 82 & $6(1)$ \\
\hline 11 & $18(1)$ & 48 & $24(4)$ & 83 & 14 \\
\hline 12 & $10(1)$ & 49 & $14(1)$ & 84 & 3 \\
\hline 13 & 1 & 50 & 18 & 87 & 2 \\
\hline 17 & 2 & 51 & 8 & 90 & $1(1)$ \\
\hline 19 & 9 & 52 & 2 & 92 & $3(1)$ \\
\hline 20 & 17 & 57 & 1 & 95 & 1 \\
\hline 21 & 19 & 58 & $5(1)$ & 98 & $3(1)$ \\
\hline 22 & 19 & 59 & $18(4)$ & 99 & 1 \\
\hline 23 & 11 & 60 & 8 & 100 & $3(1)$ \\
\hline 24 & 1 & 61 & 1 & 101 & 4 \\
\hline 25 & 3 & 62 & 1 & 102 & $6(1)$ \\
\hline 29 & $5(1)$ & 63 & $12(1)$ & 103 & 6 \\
\hline 30 & 5 & 64 & $24(3)$ & 104 & 4 \\
\hline 31 & 19 & 65 & 11 & 105 & $6(1)$ \\
\hline 32 & $14(1)$ & 66 & 12 & 106 & 2 \\
\hline 33 & $16(1)$ & 67 & $10(2)$ & 107 & 3 \\
\hline 34 & $21(1)$ & 72 & 1 & 108 & $1(1)$ \\
\hline 35 & $18(3)$ & 73 & $2(2)$ & 109 & $1(1)$ \\
\hline 36 & $17(2)$ & 74 & $1(1)$ & 111 & $5(4)$ \\
\hline 115 & 1 & 155 & 4 & & \\
\hline 117 & $2(1)$ & 156 & 2 & & \\
\hline 118 & $8(2)$ & 157 & $3(2)$ & & \\
\hline 119 & 8 & 162 & 1 & & \\
\hline 120 & 8 & & & & \\
\hline 121 & 1 & & & & \\
\hline 122 & $4(1)$ & & & & \\
\hline 123 & 3 & & & & \\
\hline 124 & 9 & & & & \\
\hline 125 & 1 & & & & \\
\hline 129 & 3 & & & & \\
\hline 133 & 1 & & & & \\
\hline 135 & 2 & & & & \\
\hline 136 & 2 & & & & \\
\hline 137 & $14(1)$ & & & & \\
\hline 138 & $19(5)$ & & & & \\
\hline 139 & $17(8)$ & & & & \\
\hline 140 & 2 & & & & \\
\hline 141 & 1 & & & & \\
\hline 142 & 2 & & & & \\
\hline 144 & $1(1)$ & & & & \\
\hline 151 & $2(1)$ & & & & \\
\hline 153 & 4 & & & & \\
\hline 154 & $7(1)$ & & & & \\
\hline
\end{tabular}

ros solamente registran una especie endémica en Oaxaca. Los géneros que contienen especies endémicas representan $42.5 \%$ de la riqueza genérica total reportada por Villaseñor et al. (2004).

$\mathrm{Al}$ evaluar la riqueza de endemismos por cuadro o celda (Figura 2), se encontró que 103 celdas (63.4\%) registran al menos una especie endémica. La celda diez registra el mayor número ( 35 especies) mientras que 22 celdas contienen una sola especie endémica. Las otras celdas con endémicas registradas reportan entre 2 y 33 especies (Cuadro 2), con una mediana igual a cuatro especies. Un total de 44 celdas registran a su vez especies exclusivas (restringidas a la celda, Cuadro 2, Figura 2); la celda 139 es la que contiene el mayor número de especies restringidas (ocho) y otras 24 registran únicamente una especie (Cuadro 2, Apéndice 1).

Las 44 celdas con especies solamente conocidas en su territorio incluyen casi toda la diversidad de endemismos del estado, pues de las 209 especies solamente seis no se han registrado en ninguna de dichas celdas (Acourtia erioloma, conocida de las celdas 45 y 60, Bidens oaxacana, presente en las celdas 104 y 121, Calea oaxacana, conocida de las celdas 124 y 142, Stevia perfoliata, restringida a las celdas 17 y 42, Tehuana calzadae, distribuida en las celdas 141, 155 y 156 y Trixis parviflora, conocida de las celdas 95 y 133). Si se añade a las 44 celdas al menos una de las celdas donde estas últimas seis especies se registran, entonces toda la diversidad de Compuestas endémicas podrían ubicarse en 50 celdas, las cuales registran una riqueza variable, desde una sola especie (siete celdas) hasta 35 (celda diez), con una mediana de seis especies por celda (Apéndice 1).

La figura 3 ilustra la distribución de los biomas en el esta-

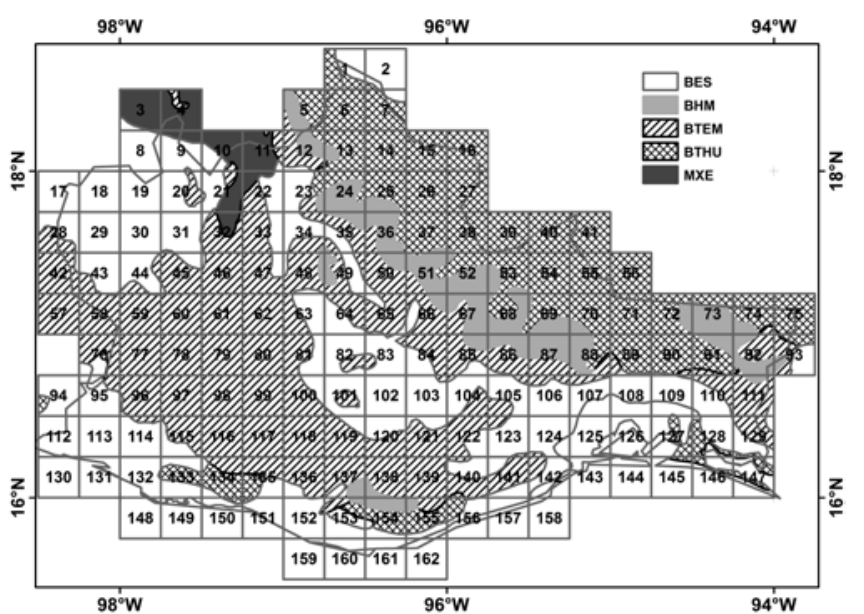

Figura 3. Principales tipos de vegetación (biomas) en el estado de Oaxaca, de acuerdo con el mapa de vegetación potencial de Rzedowski (Rzedowski, 1990). BES= Bosque estacionalmente seco, $\mathrm{BHM}=$ Bosque húmedo (mesófilo) de montaña, BTEM= Bosque templado, BTHU= bosque tropical húmedo, $\mathrm{MXE}=\mathrm{Ma}-$ torral xerófilo. 
Cuadro 3. Especies endémicas de Oaxaca y estados vecinos ( $N=209)$ con registros $(N=2,104)$ en los cinco tipos de vegetación utilizados. $\mathrm{BES}=$ Bosque estacionalmente seco, $\mathrm{BHM}=$ Bosque húmedo de montaña, BTEM = Bosque templado, BTHU = bosque tropical húmedo, $\mathrm{MXE}=$ Matorral xerófilo.

\begin{tabular}{lccc}
\hline $\begin{array}{l}\text { Tipo de } \\
\text { vegetación }\end{array}$ & Especies & $\begin{array}{c}\text { Especies } \\
\text { exclusivas } \\
\text { al bioma }\end{array}$ & $\begin{array}{c}\text { Área del bioma } \\
\text { en Oaxaca } \\
\left(\mathrm{km}^{2}\right)\end{array}$ \\
\hline BES & 116 & 21 & $35,352.8$ \\
BHM & 40 & 6 & $7,828.0$ \\
BTEM & 164 & 61 & $23,702.7$ \\
BTHU & 15 & 2 & $23,689.0$ \\
MXE & 49 & 6 & $3,122.8$ \\
\hline
\end{tabular}

do; de igual manera, el cuadro 3 indica la distribución de las especies entre los cinco biomas utilizados para el análisis. Su ubicación se realizó ya sea por su referencia directa de la etiqueta de herbario, o por su ubicación geográfica dentro de los sitios cartografiados con el mapa de vegetación potencial de Rzedowski (1990). Poco más de la mitad de las especies se registraron en más de un tipo de vegetación, pues solamente 96 de ellas (45.9\%) se conocen de uno solo; domina por el número de especies restringidas el bosque templado (61), seguido por el bosque estacionalmente seco (21 especies). Para el bosque húmedo (mesófilo) de montaña y el matorral xerófilo se reconocen seis especies exclusivas en cada uno de ellos y el bosque tropical húmedo es el que registra el menor número de especies exclusivas al bioma (dos).

\section{Discusión}

Oaxaca es el estado mexicano con la mayor riqueza total y de endemismos de Compuestas en México. Esta posición se vuelve todavía más importante al considerar al endemismo regional, es decir, al endemismo compartido con los estados vecinos. El análisis de 209 (83.9\%) de las 249 especies de Compositae conocidas de Oaxaca y estados vecinos, permite definir los patrones de endemicidad en el estado de una manera clara y confiable. Las 40 especies que no cuentan con registros en los herbarios consultados, probablemente no afectan substancialmente los resultados aquí presentados. La pobre representatividad de tales especies en las colecciones, sugieren que son especies raras en la naturaleza, por lo que el conocimiento de su distribución conocida eventualmente mostrará la presencia adicional de sitios irremplazables, o aumentará la importancia de algunos de ellos ya identificados con las especies evaluadas.

Prácticamente en todo el estado se observan especies endémicas estrictas o regionales (que extienden su presencia en los estados vecinos). Solamente las partes noreste y suroeste registran celdas sin presencia de endemismos (Figura 2). La mayor parte del territorio que no registra endemismos comprende zonas con la vegetación transformada por acti- vidades humanas, principalmente potreros para crianza de ganado o plantaciones agrícolas. Aunque no se descarta la presencia de alguna especie en tales zonas, la exploración botánica no ha sido seguramente tan interesante ni exhaustiva como en otras partes del estado.

Muchas de las especies endémicas analizadas no mostraron una alta especificidad por el hábitat, registrándose en más de un tipo de vegetación. Estudios futuros que analicen de manera más particular las diferentes comunidades vegetales aquí incluidas como un solo tipo de vegetación principal (por ejemplo las diferentes variantes de matorrales xerófilos mencionadas por Valiente-Banuet et al. 2000 para el Valle de Tehuacán-Cuicatlán), permitirán evaluar mejor la especificidad o no especificidad de las especies por una comunidad vegetal particular. La amplitud ecológica de más de la mitad de las especies endémicas, explicada por su presencia en dos biomas o más, sugiere que no son especies de origen reciente, más bien tienen la suficiente antigüedad que les permitió adaptarse a las condiciones ambientales contrastantes que determinan la presencia de estos biomas.

La superficie ocupada por los biomas en Oaxaca, es muy variable; destacan el bosque estacionalmente seco (BES), el bosque templado (BTEM) y el bosque tropical húmedo (BTHU) como los más ampliamente distribuidos (Cuadro 3). De esos tres biomas, el que mayor número de especies endémicas registra es el BTEM, superando notablemente a los otros dos biomas en el número de especies exclusivas. El menor número de endemismos en los bosques tropicales (tanto secos como húmedos) sugiere una amplia distribución geográfica de las especies adaptadas a estos ambientes, por lo que el endemismo estricto es reducido comparado con el observado en el BTEM. Las regiones montañosas son sin lugar a dudas ambientes más favorables para una diversificación por aislamiento geográfico entre las poblaciones, reflejado en el mayor número de especies exclusivas al bioma templado. El BHM y el MXE registran un porcentaje muy bajo de territorio ocupado en el estado, aunque allí se registran números importantes de Compuestas endémicas, incluyendo estrictas al bioma. Se encontró una correlación significativa aunque baja entre el área ocupada por los biomas (transformada a $\log _{10}$ ) y la riqueza de especies registrada $(R=0.46 ; P<0.05)$, lo que sugiere que la superficie del bioma, aunque importante, no es el único factor que ayuda a explicar la diversidad encontrada en su territorio.

El número de celdas con especies exclusivas (restringidas a su territorio) representa alrededor de $31.2 \%$ del territorio estatal, una superficie considerablemente grande. Sin embargo, futuros análisis en dicho territorio permitirán indudablemente reducir la superficie necesaria para realizar actividades de conservación, especialmente cuando se hagan análisis más finos en los cuadros identificados como irremplazables. Sin embargo, los resultados sugieren que la conservación de este componente de la riqueza florística estatal, podría realizarse en menor superficie que el total regis- 
trado por la Comisión Nacional para el Conocimiento y Uso de la Biodiversidad (CONABIO; Arriaga et al., 2000) en su evaluación de las regiones terrestres prioritarias. La CONABIO considera como prioritaria la conservación de $48.8 \%$ del territorio oaxaqueño en futuras estrategias de conservación. En este trabajo se identifica solamente como prioritario poco más de un tercio de la superficie estatal, lo que representa casi $18 \%$ menos de lo estimado por la CONABIO. Será igualmente importante comparar las áreas identificadas en este trabajo con las propuestas por la CONABIO, para evaluar las discrepancias en lo que estarían conservando, tal y como fue previamente subrayado por Villaseñor $e t$ al. (2004). Por ejemplo, evaluaciones preliminares indican que alrededor de 29 especies endémicas (13.9\% del total aquí analizado) no se conocen dentro de las regiones que la $\mathrm{CO}$ NABIO considera como prioritarias.

La importante correlación que existe entre la diversidad de Asteraceae y la riqueza florística total (Rzedowski 1991b; Villaseñor et al., 2007), permite suponer que los sitios relevantes por su riqueza o endemismo serán igualmente importantes en riqueza o endemicidad para otros miembros de la flora de México. Futuros análisis con otros componentes de la flora vascular en el estado, permitirán corroborar la robustez de esta correlación y así incrementar la importancia de los sitios identificados en este estudio como irremplazables o de alto valor para la conservación de la riqueza florística en el estado de Oaxaca.

\section{Agradecimientos}

Se agradece a la Comisión Nacional para el Conocimiento y Uso de la Biodiversidad (CONABIO) por el apoyo económico para el acopio y manejo de parte de la información analizada en este trabajo. Por otra parte, el Instituto de Biología de la Universidad Nacional Autónoma de México (UNAM) (Proyecto SIBA-UNIBIO) apoyó con recursos humanos que hicieron posible su realización. Un agradecimiento especial también a Enrique Ortiz por su asesoría técnica en el manejo de los datos y del sistema de información geográfica. Rosalinda Medina y Gustavo Cruz revisaron el manuscrito y contribuyeron a mejorar su contenido.

Ese trabajo se realizó en gran medida con el estudio del material depositado en el Herbario Nacional de México (MEXU) del Instituto de Biología de la UNAM. Sin embargo, se agradece a los curadores de los herbarios ENCB, IEB, OAX, SERB, TEX y XAL el permitirnos la consulta de material depositado en sus instituciones, que enriqueció substancialmente la información aquí analizada. Finalmente, M. Suárez-Mota agradece al Posgrado en Ciencias Biológicas de la UNAM. y al CONACYT (Beca 186550) por el apoyo para realizar sus estudios de postgrado; este trabajo se realizó como parte de una estancia de investigación con J. L. Villaseñor.

\section{Literatura citada}

Akeroyd J. y Synge H. 1992. Higher plant diversity. En: Groombridge, B. Ed. Global Biodiversity: Status of the Earth's Living Resources. pp. 64-87, Chapman \& Hall, Londres.

Alcántara O. y Paniagua M. 2007. Patrones de distribución y conservación de plantas endémicas. En: Luna I., Morrone J.J. y Espinosa D. Eds. Biodiversidad de la Faja Volcánica Transmexicana pp. 421-438. Universidad Nacional Autónoma de México, México, D.F.

Arriaga L., Espinoza J.M., Aguilar C., Martínez E., Gómez L. y Loa E. Coord. 2000. Regiones Terrestres Prioritarias de México. Comisión Nacional para el Conocimiento y Uso de la Biodiversidad, México, D.F.

Castillo-Campos G., Medina-Abreo M.E., Dávila-Aranda P.D. y Zavala-Hurtado J.A. 2005. Contribución al conocimiento del endemismo de la flora vascular en Veracruz, México. Acta Botanica Mexicana 73:19-57.

Cracraft J. 1982. Geographic differentiation, cladistics and vicariance biogeography: reconstructing the tempo and mode of evolution. American Zoologist 22:411-424.

Davis S.D., Heywood V.H., Herrera-MacBryde O., Villa-Lobos J. y Hamilton A.C. Eds. 1997. Centres of Plant Diversity: A Guide and Strategy for their Conservation. Volume 3. The Americas. The World Wild Fund for Nature (WWF) y The World Conservation Union (IUCN). Information Press, Oxford.

Delgadillo-M.C., Villaseñor-Rios J.L. y Dávila-Aranda P. 2003. Endemism in the Mexican flora: a comparative study in three plant groups. Annals of the Missouri Botanical Garden 90:25-34.

García-Mendoza A., Tenorio-Lezama P. y Reyes-Santiago J. 1994. El endemismo en la flora fanerogámica de la Mixteca Alta, Oaxaca-Puebla, México. Acta Botanica Mexicana 27:53-73.

González-Zamora A., Luna-Vega. I., Villaseñor J.L. y Ruiz-Jiménez C.A. 2007. Distributional patterns and conservation of species of Asteraceae (asters etc.) endemic to eastern Mexico: a panbiogeographical approach. Systematics and Biodiversity 5:135-144.

Groombridge B. Ed. 1992. Global Biodiversity. Status of the Earth's living resources. Chapman and Hall, Londres.

Henderson I.M. 1991. Biogeography without area? Australian Systematic Botany 4:59-71.

Hernández-L L. 1995. La flora endémica de la Sierra de Manantlán, Jalisco-Colima, México: observaciones preliminares. En: Vázquez-G. J.A., Cuevas-G. R., Cochrane T.S., Iltis H.H., Santana-M. F.J. y Guzmán-H. L. Eds. Flora de Manantlán, pp. 7281, Botanical Research Institute of Texas, Fort Worth.

Kier G., Mutke J., Dinerstein E., Ricketts T.H., Küper W., Kreft H. y Barthlott W. 2005. Global patterns of plant diversity and floristic knowledge. Journal of Biogeography 32:1107-1116.

Krupnick G.A. y Kress W.J. 2003. Hotspots and ecoregions: A test of conservation priorities using taxonomic data. Biodiversity and Conservation 12:2237-2253.

Méndez-Larios I., Ortiz E. y Villaseñor J.L. 2004. Las Magnoliophyta endémicas de la porción xerofítica de la provincia florística del Valle de Tehuacán-Cuicatlán, México. Anales del Instituto de Biología, Universidad Nacional Autónoma de México, Serie Botánica 75:87-104.

Mittermeier R.A. 1988. Primate diversity and the tropical forest. En: Wilson E.O. y Peter F.M. Eds. Biodiversity, pp. 145-154, National Academic Press, Washington, D.C. 
Mittermeier R.A. y Goettsch de Mittermeier C. 1992. La importancia de la diversidad biológica de México. En: Sarukhán J. y Dirzo R. Eds. México Ante los Retos de la Biodiversidad, pp. 63-73, Comisión Nacional para el Conocimiento y Uso de la Biodiversidad, México, D.F.

Morrone J.J. 1994. On the identification of areas of endemism. Systematic Biology 43:438-441.

Myers N., Mittermeier R.A., Mittermeier C.G., da Fonseca G.A.B. y Kent J. 2000. Biodiversity hotspots for conservation priorities. Nature 403:853-858.

Pressey R.L., Humphries C.J., Margules C.R., Vane-Wright R.I. y Williams P.H. 1993. Beyond opportunism: key principles for systematic reserve selection. Trends in Ecology and Evolution 8:124-128.

Reid W.V. 1998. Biodiversity hotspots. Trends in Ecology and Evolution 13:275-280.

Rosen B.R. 1988. From fossil to earth history: applied historical biogeography. En: Myers A.A. y Giller P.S. Eds. Analytical Biogeography, pp. 437-481, Chapman and Hall, Nueva York.

Rzedowski J. 1990. Vegetación potencial. IV.8.2. Atlas Nacional de México. Vol II. Escala 1:4,000,000. Instituto de Geografía, Universidad Nacional Autónoma de México, México, DF.

Rzedowski J. 1991a. El endemismo en la flora fanerogámica de México: una apreciación analítica preliminar. Acta Botanica Mexicana 15:47-64.

Rzedowski J. 1991b. Diversidad y orígenes de la flora fanerogámica de México. Acta Botanica Mexicana 14:3-21.

Turner B.L. y Nesom G.L. 1993. Biogeography, diversity, and endangered or threatened status of Mexican Asteraceae. En: Ramamoorthy T.P., Bye R., Lot A. y Fa J. Eds. Biological Diversity of Mexico: Origins and Distribution, pp. 559-575, Oxford University Press, Nueva York.

Comisión de Supervivencia de Especies de la UICN [Unión Internacional para la Conservación de la Naturaleza y de los Recursos Naturales]. 2001. Categorías y criterios de la lista Roja de la UICN. Versión 3.1. Unión Internacional para la Conservación de la Naturaleza y de los Recursos Naturales, Oxford.

Valiente-Banuet A., Casas A., Alcántara A., Dávila P., FloresHernández N., Arizmendi M. del C., Villaseñor J.L. y Ortega J. 2000. La vegetación del Valle de Tehuacán-Cuicatlán. Boletín de la Sociedad Botánica de México 67:24-74.

Vega-Aviña R., Aguiar-Hernández H., Gutiérrez-García J.A., Hernández-Vizcarra J.A., Vega-López I.F. y Villaseñor J.L. 2000. Endemismo regional presente en la flora del municipio de $\mathrm{Cu}-$ liacán, Sinaloa, México. Acta Botanica Mexicana 53:1-15.

Villaseñor J.L. 1993. La familia Asteraceae en México. Revista de la Sociedad Mexicana de Historia Natural 44: 117-124.

Villaseñor J.L. 2003. Diversidad y distribución de las Magnoliophyta de México. Interciencia 28:160-167.

Villaseñor J.L. y Elias T.S. 1995. Análisis de especies endémicas para identificar áreas de protección en Baja California, México. En: Linares E., Dávila P., Chiang F., Bye R. y Elias T.S. Eds. Conservación de Plantas en Peligro de Extinción: Diferentes Enfoques, pp. 43-50, Instituto de Biología, Universidad Nacional Autónoma de México. México, D.F.

Villaseñor J.L., Delgadillo C. y Ortiz E. 2006. Biodiversity hotspots from a multigroup perspective: Mosses and Senecios in the Transmexican Volcanic Belt. Biodiversity and Conservation 15:4045-4058.

Villaseñor J.L. y Ortiz E. 2007. La familia Asteraceae. En: Luna I., Morrone J.J. y Espinosa D. Eds. Biodiversidad de la Faja Volcánica Transmexicana. pp. 289-310, Universidad Nacional Autónoma de México, México, D.F.

Villaseñor J.L., Ortiz E. y Juárez V. 2004. Asteráceas. En: GarcíaMendoza A.J., Ordoñez M. J. y Briones-Salas M. Eds. Biodiversidad de Oaxaca, pp. 177-192, Instituto de Biología, Universidad Nacional Autónoma de México, Fondo Oaxaqueño para la Conservación de la Naturaleza y World Wildlife Found, México, D.F.

Villaseñor J.L., Maeda P., Rosell J.A. y Ortiz E. 2007. Plant families as predictors of plant biodiversity in Mexico. Diversity and Distributions 13:871-876. 
Apéndice 1. Especies endémicas de México presentes en Oaxaca y estados vecinos con registros georreferenciados (Figura 1). Después del nombre, se indica el o los estados donde se documenta su presencia, el tipo de vegetación y las celdas en que Oaxaca fue dividido donde han sido registradas. $C H I S=$ Chiapas, $G R O=$ Guerrero, $\mathrm{OAX}=$ Oaxaca, $\mathrm{PUE}=$ Puebla, $\mathrm{VER}=$ Veracruz, $\mathrm{BES}=$ Bosque estacionalmente seco, $\mathrm{BHM}=$ Bosque húmedo (mesófilo) de montaña, BTEM = Bosque templado, BTHU = Bosque tropical húmedo, MXE = Matorral xerófilo.

Achyrocline oaxacana G.L. Nesom OAX; BTEM; 35

Acourtia ciprianoi Panero \& Villaseñor OAX; BES; 157

Acourtia discolor Rzed. OAX; BES, BTEM; 31, 34, 43, 48

Acourtia erioloma (S.F. Blake) Reveal \& R.M. King OAX; BTEM; 45, 60

Acourtia lobulata (Bacig.) Reveal \& R.M. King OAX, PUE; BES, BTEM, MXE; 9, 10, 48, 50

Acourtia oaxacana L. Cabrera OAX; BES; 102

Acourtia ovatifolia L. Cabrera GRO, OAX, PUE; BES, BTEM; 4, 31, 48

Acourtia scapiformis (Bacig.) B.L. Turner OAX, PUE; BES, BTEM, MXE; 4, 9, 10, 19, 21, 22, 29, 31, 33, 34, 43, 44, 47, 48

Acourtia souleana B.L. Turner OAX, PUE; BES, MXE; 9, 10, 19

Acourtia tenoriensis B.L. Turner OAX, PUE; BES, BTEM, MXE; 9, 20, 21, 22, 46

Acourtia umbratilis (B.L. Rob. \& Greenm.) B.L. Turner OAX, PUE; BES, MXE; 34

Ageratina aegirophylla (B.L. Rob.) R.M. King \& H. Rob. OAX; BES; 63

Ageratina chimalapana B.L. Turner OAX; BTEM, 92

Ageratina collodes (B.L. Rob. \& Greenm.) R.M. King \& H. Rob. OAX; BES, BHM, BTEM; 19, 23, 35, 47, 48, 63, 64, 65, 66, 83, $84,101,120,122$

Ageratina cruzii B.L. Turner OAX; BTEM; 46

Ageratina desquamans (B.L. Rob.) R.M. King \& H. Rob. OAX; BTEM; 81

Ageratina etlensis (B.L. Rob.) R.M. King \& H. Rob. OAX; BTEM; 48

Ageratina glauca (Sch. Bip. ex Klatt) R.M. King \& H. Rob. OAX, VER; BHM, BTEM; 35, 36, 66

Ageratina humochica B.L. Turner OAX; BTEM; 36

Ageratina juxtlahuacensis Panero \& Villaseñor OAX; BES; 43

Ageratina kochiana B.L. Turner OAX; BTEM; 46

Ageratina macdonaldii B.L. Turner OAX; BTEM; 139

Ageratina mayajana B.L. Turner OAX; BTEM; 111

Ageratina miahuatlana B.L. Turner OAX; BTEM; 118, 138, 139

Ageratina pendula Panero \& Villaseñor OAX; BTEM; 59

Ageratina peracuminata R.M. King \& H. Rob. OAX; BTEM; 117

Ageratina pochutlana B.L. Turner OAX; BHM, BTEM, 138155

Ageratina rupicola (B.L. Rob. \& Greenm.) R.M. King \& H. Rob. OAX; BTEM; 20, 46, 81

Ageratina seleri B.L. Turner OAX; BES, BTEM; 19, 46, 49, 64, 65, 83, 84, 101

Ageratina sousae B.L. Turner OAX; BES, BTEM; 11, 12, 33, 34, 46, 59

Ageratum albidum (DC.) Hemsl. OAX; BES, BTEM; 19, 22, 47, 48, 49, 50, 63, 64, 65, 82, 104, 119

Ageratum tehuacanum R.M. King \& H. Rob. OAX, PUE, VER; BES, BTEM, MXE; 4, 9, 10, 11, 20, 21, 22, 23, 31, 34, 48

Alepidocline macdonaldana B.L. Turner OAX; BTEM; 139

Alepidocline trifida (J.J. Fay) B.L. Turner OAX; BES, BTEM; 22, 48, 50, 81, 120, 135, 137

Aphanactis macdonaldii B.L. Turner OAX; BTEM; 139

Archibaccharis albescens (J.D. Jacks.) G.L. Nesom OAX; BTEM; 81, 138

Archibaccharis campii S.F. Blake OAX; BHM, BTEM; 67

Archibaccharis macdonaldii G.L. Nesom OAX; BTEM; 139

Archibaccharis nephocephala G.L. Nesom OAX; BTEM; 138

Archibaccharis pringlei (Greenm.) S.F. Blake OAX; BTEM; 81

Archibaccharis trichotoma (Klatt) G.L. Nesom OAX; BHM, 67

Axiniphyllum corymbosum Benth. GRO, OAX; BES, BTEM; 42, 43, 44, 50, 59, 135

Axiniphyllum scabrum (Zucc.) S.F. Blake OAX; BES, BTEM; 48, 62, 63, 64, 81

Aztecaster pyramidatus (B.L. Rob. \& Greenm.) G.L. Nesom OAX; BES, BTEM, BTHU; 9, 21, 24, 29, 32, 33, 44, 60, 63, 64, 82, 83, 84, 119, 137

Baccharis glandulifera G.L. Nesom CHIS, OAX; BES, BTEM; 31, 35, 64, 118, 138 
Baccharis mexicana Cuatrec. GRO, OAX, PUE, VER; BES, BHM, BTEM, MXE; 4, 9, 10, 11, 20, 21, 23, 31, 33, 34, 35, 44, 45, 46, 59, 60, 63, 64, 65, 83, 101, 105, 120, 137

Bartlettina calderonii (B.L. Turner) B.L. Turner OAX; BHM; 36

Bartlettina constipatiflora (Klatt) R.M. King \& H. Rob. CHIS, OAX; BHM, BTEM, BTHU; 35, 66, 67

Bartlettina hintonii R.M. King \& H. Rob. GRO, OAX; BES, BTEM; 43

Bartlettina macdougallii R.M. King \& H. Rob. OAX, PUE; BHM, BTEM, BTHU; 12, 36, 51

Bidens oaxacana Melchert OAX; BES; 104, 121

Bidens sharpii (Sherff) Melchert GRO, OAX, PUE; BES, BTEM; 9, 20, 30, 31, 36

Brickellia problematica B.L. Turner OAX, PUE; BES, BTEM, MXE; 9, 10, 11, 20, 21, 32, 46

Calea megacephala B.L. Rob. \& Greenm. CHIS, OAX; BES, BHM, BTEM, BTHU; 129, 139, 153

Calea oaxacana (B.L. Turner) B.L. Turner OAX; BES, MXE; 124, 142

Chaptalia pringlei Greene OAX, PUE; BES, BTEM, MXE; 4, 9, 10, 21, 22, 31, 33, 43, 44, 47

Chionolaena aecidiocephala (Grierson) Anderb. \& S.E. Freire CHIS, OAX; BTEM; 35

Chionolaena eleagnoides Klatt OAX; BHM, BTEM; 35, 36

Chionolaena macdonaldii (G.L. Nesom) G.L. Nesom OAX; BTEM; 139

Chromolaena breedlovei R.M. King \& H. Rob. CHIS, OAX; BHM, BTEM; 111

Chromolaena guiengolense (L. Torres \& Villaseñor) B.L. Turner OAX; BES, BTEM; 29, 59, 124

Chrysanthellum perennans B.L. Turner OAX; MXE; 109

Chrysanthellum pilzii Strother OAX; BES; 151

Cirsium imbricatum (B.L. Rob. \& Greenm.) Petr. OAX; BTEM; 81

Coreopsis oaxacensis B.L. Turner OAX, PUE; BES, BTEM; 9, 20

Cosmos juxtlahuacensis Panero \& Villaseñor OAX; BES; 43

Cosmos nelsonii B.L. Rob. \& Fernald OAX; BTEM; 79

Cosmos sherffii Melchert OAX; BTEM; 47, 48

Critoniopsis macvaughii (S.B. Jones) H. Rob. GRO, OAX, PUE; BES, BTEM, MXE; 9, 10, 19, 30, 31, 32, 43, 46, 47, 48, 59

Critoniopsis tarchonanthifolia (DC.) H. Rob. OAX; BES, BTEM; 43, 64, 65, 81

Dahlia apiculata (Sherff) P.D. Sørensen PUE; BES, MXE; 9, 10

Dahlia macdougallii Sherff OAX; BES, BTEM, MXE; 9, 124, 140

Dahlia pteropoda Sherff OAX, PUE; BES, MXE; 10, 11

Dahlia tenuis B.L. Rob. \& Greenm. OAX; BTEM; 63, 81

Desmanthodium perfoliatum Benth. CHIS, GRO, OAX; BES, BHM, BTEM, BTHU; 44, 49, 50, 51, 64, 66, 81, 105, 137, 140, 153

Digitacalia napeifolia (DC.) Pippen OAX; BES, BTEM; 43, 49, 50, 64, 81, 83

Erigeron oaxacanus Greenm. OAX; BES; 144

Erigeron quiexobrensis G.L. Nesom OAX; BTEM; 139

Eupatoriastrum chlorostylum B.L. Turner OAX; BES; 154

Flaveria cronquistii A.M. Powell OAX, PUE; BTEM, MXE; 11

Flaveria kochiana B.L. Turner OAX; BES; 29

Flaveria ramosissima Klatt OAX, PUE; BES, BTEM, MXE; 9, 11, 12, 22

Flaveria vaginata B.L. Rob. \& Greenm. OAX; BES, BTEM; 19, 29, 30, 32

Fleischmannia gonzalezii (B.L. Rob.) R.M. King \& H. Rob. OAX; BTEM; 33

Florestina platyphylla (B.L. Rob. \& Greenm.) B.L. Rob. \& Greenm. OAX; BES, BTEM; 50, 63, 64, 81, 83, 101, 102, 103, 104,120

Florestina purpurea (Brandegee) Rydb. OAX, PUE; BES, BTEM, MXE; 9, 10, 32, 44, 45, 60, 83

Florestina simplicifolia B.L. Turner OAX, PUE; BES, BTEM, MXE; 3, 4, 9, 10, 11, 22, 23, 31, 34,83

Flourensia collodes (Greenm.) S.F. Blake CHIS, OAX; BES, BTHU; 102, 124, 151

Flourensia glutinosa (B.L. Rob. \& Greenm.) S.F. Blake GRO, OAX, PUE; BES, BTEM, MXE; 3102234

Gnaphalium oblanceolatum Greenm. OAX; BES; 64

Gochnatia smithii B.L. Rob. \& Greenm. OAX; BES, BTEM, MXE; 10, 22, 23, 33, 34, 98, 103

Grindelia oaxacana G.L. Nesom OAX; BES; 48

Gymnolaena oaxacana (Greenm.) Rydb OAX, PUE; BES, BHM, BTEM, MXE; 4, 9, 10, 11, 22, 23, 33, 34, 47, 48, 59 
Apéndice 1. Continuación

Gymnolaena serratifolia (DC.) Rydb OAX; BES, BTEM; 67, 83

Heliomeris obscura (S.F. Blake) Cockerell OAX, PUE; BES, MXE; 9, 10, 21

Hidalgoa uspanapa B.L. Turner CHIS, VER; BTHU; 74

Hieracium macdonaldii Beaman \& B.L. Turner OAX; BTEM; 122, 138, 139

Hofmeisteria malvifolia (B.L. Rob. \& Greenm.) B.L. Turner OAX, PUE; BES, MXE; 10, 11, 23, 33, 34

Jefea pringlei (Greenm.) Strother OAX, PUE; BES, BTEM, MXE; 4, 9, 10, 21, 34

Koanophyllon coixtlahuacum B.L. Turner OAX; MXE; 10

Koanophyllon revealii B.L. Turner GRO; BTEM

Kyrsteniopsis dibollii R.M. King \& H. Rob. PUE, VER; BTEM, MXE; 11

Kyrsteniopsis eriocarpa (B.L. Rob. \& Greenm.) B.L. Turner OAX, PUE; BES, BTEM; 19, 22, 34

Leiboldia guerreroana (S.B. Jones) H. Rob. GRO, OAX; BES, BTEM, 59

Lepidonia jonesii (B.L. Turner) H. Rob. \& V.A. Funk OAX; BHM, BTEM, BTHU, MXE; 12, 13, 25, 35, 36, 46, 49, 50, 51, 52, $66,67,105,125$

Macvaughiella mexicana (Sch. Bip.) R.M. King \& H. Rob. VER; BES, BHM, BTHU

Melampodium glabribracteatum Stuessy OAX; BES; 82

Melampodium mimulifolium B.L. Rob. OAX; BHM, BTEM; 65, 66, 138

Melampodium northingtonii B.L. Turner OAX; BES; 153, 154

Melampodium pringlei B.L. Rob. OAX; BTEM, 48

Mikania pooleana W.C. Holmes \& Vodopich OAX; BTEM, 35

Mikania tehuacanensis W.C. Holmes OAX; BTEM, 12

Montanoa liebmannii (Sch. Bip.) S.F. Blake OAX; BES, BTEM; 44, 47, 48, 60, 81, 83, 137

Neurolaena fulva B.L. Turner CHIS, OAX; BES, BHM, BTEM; 92, 111

Neurolaena lamina B.L. Turner OAX, VER; BTHU; 90

Neurolaena macrocephala Sch. Bip. ex Hemsl. OAX, VER; BES, BHM, BTHU; 1, 36, 72

Neurolaena oaxacana B.L. Turner OAX; BHM, BTEM; 36, 49, 50, 67

Oteiza mixtecana Villaseñor \& Panero OAX; BES, BTEM; 20, 31, 46

Oteiza scandens Panero \& Villaseñor OAX; BTEM; 138

Oxylobus oaxacanus S.F. Blake OAX; BHM, BTEM; 12, 35, 67

Paneroa stachyofolia (B.L. Rob.) E.E. Schill. OAX; BES, BTEM; 31, 32, 33, 44, 60, 64

Pectis canescens Kunth GRO, OAX; BES 157

Pentacalia venturae (T.M. Barkley) C. Jeffrey CHIS, OAX, VER; BES, BHM, BTEM; 11, 66

Perymenium discolor Schrad. OAX, PUE; BES, BTEM, MXE; 4, 9, 10, 21, 22, 31, 32, 35, 36, 43, 46, 49, 50, 63, 64, 65, 81, 83

Perymenium oaxacanum B.L. Turner OAX; BTEM; 47, 138

Perymenium ovatum Brandegee PUE; MXE; 9,10

Perymenium sedasanum J.J. Fay OAX; BTEM; 48

Podachaenium chimalapanum B.L. Turner OAX; BHM; 73

Psacaliopsis macdonaldii (B.L. Turner) C. Jeffrey OAX; BTEM; 139

Psacaliopsis purpusii (Greenm.) H. Rob. \& Brettell OAX, PUE; BES, BTEM, MXE; 9, 10, 20, 21

Psacalium beamanii H. Rob. OAX; BTEM; 49

Psacalium calvum (Brandegee) Pippen PUE; BES; 9

Psacalium guerreroanum B.L. Turner GRO, OAX; BES, BTEM; 20, 44, 59

Psacalium hintoniorum B.L. Turner OAX; BTEM; 118

Psacalium nelsonii Rydb. OAX; BTEM; 81, 119, 139

Psacalium paucicapitatum (B.L. Rob. \& Greenm.) H. Rob. \& Brettell OAX; BES, BTEM; 20, 22, 31, 33, 44, 60, 78, 81

Psacalium purpusii (Greenm.) H. Rob. \& Brettell OAX, PUE; BES, BTEM, MXE; 10, 20, 22, 31, 44,48

Psacalium schillingii Panero \& Villaseñor OAX; BES, BTEM; 43, 44

Roldana anisophylla (Klatt) Funston OAX; BHM, BTEM; 25, 35, 36, 49, 50, 67, 137

Roldana calzadana B.L. Turner OAX; BTEM; 43

Roldana cordovensis (Hemsl.) H. Rob. \& Brettell CHIS, OAX, PUE, VER; BES, BHM, BTEM, BTHU; 12, 51, 63, 136, 153

Roldana eriophylla (Greenm.) H. Rob. \& Brettell CHIS, GRO, OAX, PUE; BES, BHM, BTEM, BTHU, MXE; 21, 23, 31, 34, 44, 64, 82, 83, 103, 107, 119, 124, 155 
Apéndice 1. Continuación

Roldana heteroidea (Klatt) H. Rob. \& Brettell OAX; BTEM; 81, 137

Roldana mixtecana Panero \& Villaseñor OAX; BES, BTEM; 43

Roldana oaxacana (Hemsl.) H. Rob. \& Brettell OAX, PUE, VER; BES, BHM, BTEM, MXE; 9, 10, 11, 21, 22, 25, 31, 32, 33, $34,35,36,43,44,46,47,49,50,51,52,58,59,63,64,65,66,67,77,81,98,100,107,117,118,119,120,123,124$, $137,138,139$

Roldana subcymosa H. Rob. CHIS, OAX; BTEM; 81, 137, 139

Roldana uxordecora Quedensley \& Villaseñor OAX; BTEM; 137

Sanvitalia fruticosa HemsI. PUE; MXE; 4, 10

Schistocarpha calzadana B.L. Turner GRO, OAX; BTEM; 58

Senecio bracteatus Klatt GRO, OAX; BES, BHM, BTEM; 9, 10, 22, 23, 33, 36, 43, 45, 48, 49, 50, 57, 58, 59, 60, 64, 65, 99, $118,119,137,138,139,154$

Senecio conzattii Greenm. OAX, PUE; BES, BHM, BTEM, BTHU, MXE; 10, 11, 12, 20, 22, 23, 34, 35, 36, 49, 50, 64, 65, $66,77,83,118$

Senecio polypodioides (Greene) Greene GRO, OAX; BES, BHM, BTEM; 35, 36, 43, 49, 81, 120, 137, 138,154

Senecio subauriculatus Greenm. OAX; BTEM; 120, 137, 138, 139

Sigesbeckia repens B.L. Rob. \& Greenm. OAX; BTEM, 81, 118, 119, 138

Simsia sylvicola Panero \& E.E. Schill. OAX; BTEM; 100

Simsia villasenorii D.M. Spooner CHIS, OAX; BES, BTEM; 103, 124, 129, 157

Sinclairia ismaelis Panero \& Villaseñor OAX; BHM; 138

Stachycephalum mexicanum Sch. Bip. ex Benth. OAX; BES; 64

Stevia caltepecana B.L. Turner PUE; BES; 9

Stevia calzadana B.L. Turner GRO, OAX; BTEM; 43, 58

Stevia crassifolia Soejima \& Yahara OAX; BTEM; 64

Stevia cruzii Grashoff OAX, PUE; BES, BTEM, MXE; 21, 33

Stevia decumbens (B.L. Rob. \& Greenm.) Greene OAX, PUE; BES, BTEM, MXE; 34, 46, 48, 64

Stevia ecatepecana Soejima, Yahara \& K. Watanabe OAX; BES; 122

Stevia ephemera Grashoff GRO, OAX; BES, BTEM; 21, 31, 32, 34, 46, 59, 61

Stevia liebmannii Sch. Bip. ex Klatt OAX; BTEM; 34, 47, 48, 137

Stevia oaxacana Soejima \& Yahara OAX; BTEM; 118

Stevia perfoliata Cronquist GRO, OAX; BES, BTEM; 17, 42

Stevia quiexobra B.L. Turner OAX; BTEM; 139

Stevia revoluta B.L. Rob. OAX, PUE; BES, BTEM, MXE; 9, 20, 21

Stramentopappus pooleae (B.L. Turner) H. Rob. \& V.A. Funk OAX; BES, BHM, BTEM; 34, 35, 36, 50, 51, 66, 67

Tagetes linifolia Seaton OAX, PUE, VER; BES, BHM, BTEM, MXE; 32

Tagetes oaxacana B.L. Turner OAX; BTEM; 59

Tehuana calzadae Panero \& Villaseñor OAX; BES, BTEM; 141, 155, 156

Telanthophora liebmannii (Buchinger ex Klatt) H. Rob. \& Brettell OAX; BHM, BTEM; 35, 36, 50, 51, 64, 66

Tetrachyron brandegeei (Greenm.) Wussow \& Urbatsch OAX, PUE; BES, BTEM, MXE; 9, 10, 11, 20, 33

Tetrachyron chimalapanum B.L. Turner OAX, VER; BTEM; 111

Tetrachyron manicatum Schltdl. OAX, VER; BES, BHM, BTEM, BTHU, MXE; 1, 5, 11, 12

Tetrachyron torresii B.L. Turner OAX; BTEM; 105

Thymophylla aurantiaca (Brandegee) Rydb. OAX, PUE; BES, BTEM, MXE; 9, 10, 11, 20, 21, 32

Tithonia pedunculata Cronquist OAX; BES, BTEM; 87, 103, 104, 106, 122, 123

Tridax luisana Brandegee OAX, PUE; BES, BTEM, MXE; 9, 10, 21, 32

Tridax oaxacana B.L. Turner OAX; BTEM; 98

Tridax serboana B.L. Turner OAX; BTEM; 78

Trixis parviflora C.E. Anderson OAX; BES, BTEM; 95, 133

Trixis silvatica B.L. Rob. \& Greenm. OAX; BES, BHM, BTEM, MXE; 30, 102, 105, 106, 107, 123, 124, 142, 154, 155, 156, 162

Verbesina auriculata DC. OAX, PUE; BES, BTEM; 44, 102, 124

Verbesina calzadae Panero \& Villaseñor OAX; BES; 43

Verbesina crassipes B.L. Rob. \& Greenm. OAX; BTEM; 32, 65, 80 
Apéndice 1. Continuación

Verbesina fayii B.L. Turner OAX; BTEM, 59, 138

Verbesina juxtlahuacensis Panero \& Villaseñor OAX; BES; 43

Verbesina macdonaldii B.L. Turner OAX; BHM, BTEM; 120, 138, 139, 154

Verbesina miahuatlana B.L. Turner OAX; BTEM, 138

Verbesina mixtecana Brandegee PUE; MXE; 10

Verbesina neriifolia Hemsl. CHIS, OAX; BES, BHM, BTEM, BTHU; 51, 66, 87, 92, 105, 129

Verbesina pellucida Villaseñor \& Panero OAX; BES; 108

Verbesina petrophila Brandegee PUE; MXE; 10

Verbesina reyesii Panero \& Villaseñor OAX; BTEM; 46

Verbesina scabrida Rzed. GRO, OAX; BES, BHM, BTEM; 59, 115, 136, 154

Verbesina sericea Kunth \& C.D. Bouché OAX; BES, BTEM; 47, 48, 49, 50, 63, 64, 82, 83, 100, 102, 119

Verbesina villasenorii B.L. Turner OAX; BTEM; 59

Vernonanthura cronquistii (S.B. Jones) H. Rob. GRO, OAX; BES, BTEM; 58, 59

Vernonia wendtiana B.L. Turner OAX; BHM; 73

Viguiera benziorum B.L. Turner OAX; BTEM; 78

Viguiera insignis Miranda GRO, OAX, PUE; BES, BTEM, MXE; 3, 10, 17, 19, 44

Viguiera neocronquistii B.L. Turner OAX; BHM; 138

Viguiera ovata (A. Gray) S.F. Blake CHIS, OAX; BES, BTEM; 43, 64, 81

Viguiera pinnatilobata (Sch. Bip.) S.F. Blake OAX, PUE; BES, BTEM, MXE; 9, 10, 11, 22, 23, 34, 48, 50, 82, 103

Viguiera rhombifolia (B.L. Rob. \& Greenm.) S.F. Blake OAX, PUE; BES, BTEM; 4, 21, 30, 33, 46, 47, 48, 59

Wedelia purpurea (Greenm.) B.L. Turner CHIS, GRO, OAX; BES, BHM, BTEM; 111

Zaluzania subcordata W.M. Sharp OAX, PUE, VER; BES, BTEM, MXE; 9, 10, 20, 31, 32 